\title{
Service-oriented Architecture for Household Energy Management
}

\author{
Marten van Sinderen ${ }^{1}$, Martijn Warnier ${ }^{2}$, and Boris Shishkov ${ }^{2,3}$ \\ ${ }^{1}$ University of Twente, Enschede, The Netherlands \\ ${ }^{2}$ Delft University of Technology, Delft, The Netherlands \\ ${ }^{3}$ IICREST, Sofia, Bulgaria \\ m.j.vansinderen@utwente.nl, \{M.E.Warnier, B.B.Shishkov\}@tudelft.nl
}

\begin{abstract}
Recent advances in pervasive computing foster automated systems to support household energy management. Smart devices can be used to monitor both the consumption of household appliances and the presence and activity of people in the household. Based on monitoring results, intelligent feedback to residents and intelligent control of appliances is possible. In this paper we present a service-oriented architecture for household energy management in order to cope with interoperability and flexibility issues that exist in the home environment. We also propose a home service bus that realizes core properties of the service-oriented architecture, and thus facilitates integration of existing solutions and development of new applications.
\end{abstract}

Keywords: Household energy management; energy consumption monitoring; context monitoring; home service bus; service-oriented architecture (SOA).

\section{Introduction}

Technological developments such as home networking, sensor networks and pervasive computing $[45,57,21,44]$ have provided many possibilities to apply ICT in the home environment to support needs and desires of the residents. In this paper we focus on ICT support for household energy management, however we explicitly consider extension of proposed solutions to other application areas.

This paper builds on the work presented in [48] and is a companion paper of [52]. In [48] the authors present a new approach for household energy management. The approach consists of an explanation of how results from autonomic and context-aware computing can be applied to contribute to the realization of certain defined objectives for energy management. This leads to the identification of behavioral patterns and key components, constituting a basic architecture for household energy management.

The energy management system has to base its management decisions on information gathered from controlled appliances and from context sources. The representation of this information as knowledge with which the system can adaptively reason is outside the scope of [48] but is further explored in [52].

This paper extends [48] in yet another direction. It reconsiders the basic architecture and extends this architecture based on requirements such as 
interoperability and flexibility. The resulting architecture is service-oriented, and features programmable process and decision services for monitoring and controlling appliances. We claim that the architecture is generic enough to be specialized and extended to support other home/household application areas such as home automation and homecare. For this purpose we propose a home service bus as a common serviceoriented backbone.

The contribution of the paper lies in the exploration of service-oriented architecture solutions for smart home applications in general and household energy management in particular. We discuss several architectural patterns that exploit the service-oriented paradigm and we show how heterogeneous technology solutions can be integrated.

The remainder of this paper is structured as follows: Section 2 present the motivation for working on household energy management and presents related work. Section 3 summarizes the basic system architecture introduced in [48]. Section 4 discusses a basic service-oriented architecture for household energy management system. This architecture is derived from the basic system architecture, considering interoperability and flexibility requirements. This section also briefly explores possibilities to extend the architecture for multi-level energy management, which will be relevant if household energy management systems are being federated. Section 5 considers high level goals that drive the decision-making behavior of the household energy management system. Section 6 discusses the possibility of employing a home service bus to realize key properties of the service-oriented architecture. Finally, Section 7 presents our main conclusions and outlook.

\section{Motivation and Related Work}

The motivation to work on household energy management emerges from the general concerns about environmental load of consumption, and resulting policies to achieve energy preservation, i.e. using energy efficiently and reducing energy consumption [24]. We briefly discuss efforts and methods to achieve energy preservation in the home and then turn to technology-based solutions that can lead to energy efficient smart homes.

\subsection{Energy Conservation}

Household energy use accounts for a significant portion of the total energy footprint of OECD countries, and reducing the energy consumption and emissions from homes and communities is therefore considered a cost effective way to contribute to a sustainable society [16]. Three general routes have been identified for reducing energy consumption in the residential sector [55]:

1. Promote low-energy buildings;

2. Promote energy-efficient domestic equipment;

3. Promote energy conscious behavior of end-users.

We still know little about the behavior of end-users: how they manage their energy consumption, and how they can be motivated to change to a more energy conscious 
behavior [7]. A review on the effectiveness of interventions showed that rewards are most effective in achieving energy conservation, although the effect may be shortlived, followed by frequent feedback [2]. A study on feedback revealed the key features for being successful: feedback should be frequent, persistent and appliancespecific, in addition to being presented in a clear and appealing way [23].

Encouraging energy conservation in the home is not easy, primarily because the impact and amount of energy use are not readily apparent. The following measures are considered in order to improve on this situation [5]:

- Energy labeling: Providing energy labels for white goods and appliances is wellestablished, and is an important point-of-sale source of consumer information on comparative lifecycle energy use.

- Immediate feedback: Energy monitoring using smart meters and advanced user interfaces is an obvious point-of-use source of consumer information on daily energy use. Some authors have claimed up to $20 \%$ reduction in total domestic energy use, the precise amount depending on the content and method of the feedback [55, 11, 23]. Recent work [12] indicates that actual implementation of smart metering at the household level is still in its infancy and hard evidence on what can be achieved by it is scarce.

- Carbon labeling: Instead of providing information on the lifecycle energy use of equipment, it would be more appropriate to give information on the total embodied energy and carbon content of a product. This so-called carbon labeling would entail consideration of energy required and emissions induced for production, packaging, transportation, storage as well as lifecycle use. Although attractive form an energy conservation point of view, mandatory carbon labeling is probably hard to achieve because of opposition from industry groups [5].

Household energy consumption varies dramatically with time of day and time of year [55]. The electricity system would be able to maximize its efficiency if smoother or more predictable electrical demand profiles are available. For this reason, interest areas also include:

- Energy use components and patterns: Domestic electricity use is dominated by heating our house and our water [16]. A report of the US Department of Energy [19] gave the following contributions to household energy use: appliances $30 \%$, air condition, heating and ventilation $31 \%$, and water heating $9 \%$. Components of energy use may be roughly classified as predictable, moderately predictable and unpredictable [55]. Furthermore, energy use patterns are different for different types of equipment (e.g., for lighting, cooking, heating), and overall energy use patterns are different for different household compositions (e.g., single, couple, family with children) [30]. Despite these differences, clear peak times of energy use exist, which place high demands on the energy grid. The possibilities to use or change energy use patterns in order to smoothen energy use are limited. Most promising are multi-user systems [30] which can rely on the more predictable behavior of a community, compensate for irregular behavior of individuals, and time shift individual demands in order to smoothen the overall consumption peaks of the community. 
- Green micro-generation: Domestic generation of energy with renewable sources, such as sun, wind and thermal heat, has many advantages. Heat and electricity can be produced locally, which may be used for immediate daily energy needs or locally captured and used during (peak) energy demand periods. Alternatively, surplus power from micro-generation may be sold to the local power company and injected in the grid [15]. Use of fossil fuels for centralized energy production is thus decreased, losses due to centralized production and transportation over the grid are reduced, and strain on the energy grid during peak periods is lowered. Besides the environmental benefits, consumers also have the financial gain of saving on energy costs or selling to the power company. However, despite the potential benefits, there are still substantial barriers to the large-scale use of micro-generation [4].

\subsection{Pervasive Computing and Smart Homes}

Household energy management has been addressed by several researchers in the smart homes domain. The smart homes concept, also called home automation or domotics, is about the integrated application of technologies that help to improve the quality of life of the residents of the home [31, 40]. Energy management is just one of the potential application areas of smart homes, next to controlling household appliances and multi-media equipment, providing assistance to elderly and disabled, and increasing safety and security. The technologies considered here have evolved from advances in pervasive computing, also known as ubiquitous computing or ambient intelligence [53, 44, 1]. Important topics in this domain, which also have high relevance to household energy management, include:

- Sensors and actuators: RFID and other pervasive technologies [21, 3] enabled the development of miniature sensors and actuators with communication capabilities. These devices can be embedded in our environment, and integrated in equipment, daily objects, cloths or even our body. Thanks to their communication capabilities, they can be identified, traced and sometimes remotely controlled using other computers.

- Context-awareness: Sensors that measure physical properties of an environment can communicate sensor data across the home network. Based on aggregation of such data and logical inference, so-called context information can be derived which is relevant to end-user applications [13]. The latter may adapt their behavior to be more efficient or effective, given the perceived or predicted context status of their environment. In particular, applications can be more useful to end-users if provided services can be personalized to the changing situation and corresponding needs of individual users [39, 17]. Personalization can be in terms of functionality or content provided by a service, or in terms of time, form or location of its delivery. Use of automatically derived context information, instead of explicit user input, ensures that the personalization is non-intrusive to the end-user. User patterns or habits may be learned by analyzing historical context information obtained over longer time periods, and 
matching real-time context information with these patterns allows pro-active context-aware behavior [49].

- Network infrastructure and interoperability: A smart home will comprise various components, which may have been acquired at different times, supplied by different vendors, and developed by different manufacturers. This potential heterogeneity of components, combined with the diversity of physical media solutions for home environments, poses a serious interconnection and interoperability problem [18, 28]. Most existing houses were built without communication infrastructure, so they have to be retrofitted with new network technology. Powerline solutions and wireless networks present retrofits which are easy to install, flexible and relatively cheap, compared to dedicated wiring, although they are limited with respect to bandwidth and reliability. On top of low-level protocols for network connectivity, several protocols exist to communicate content and control devices. These protocols can be grouped in application areas, such as entertainment, lightning, appliances, and climate control, effectively forming different home networks [50]. In order to achieve integration of these different home networks and associated applications, middleware and service-oriented approaches have been proposed [50, 56].

- Smart applications: The previous topics are all necessary to enable the raison d'être of smart homes, namely to provide smart applications. Smart applications can increase comfort, interest or fun for users, without requiring explicit management or control from users. This means that a smart home system should be able to monitor itself or its environment, interpret monitoring results with respect to user goals, and decide on actions that would contribute to attaining the user goals. Several solutions exist for learning, knowledge representation, knowledge reasoning, and decision making, which have been applied in various smart applications, including inhabitant-aware home automation [49, 8], load balancing on the electric grid [26], reactive and proactive household energy control [34], and location aware resource management [43].

Despite the considerable progress made in pervasive computing, smart home solutions have not yet been massively deployed. Main reasons are complex installation and interfaces paired with lack of interoperability [28]. Moreover, many proposed smart home systems enable control over devices and artifacts, rather than promoting control over the lives of people. The latter has been identified as a key factor of success [14]. Such control over lives may be used to pursue 'calm living', although [42] claims that success of pervasive computing is more likely if it is used to engage people, to provoke them to be creative and active.

An important trigger for starting a smart home revolution may come from government policies to install smart energy meters into the home nationwide [16]. From this situation, energy conservation in the home and energy selling to the grid may evolve as potential killer applications. 


\section{Basic System Architecture}

Figure 1 illustrates the basic architecture for household energy management, as introduced in [48]. It shows the controlled domain with household appliances which are being monitored and/or which can be controlled concerning energy consumption. For this purpose, each appliance is instrumented with sensors and/or actuators. The controlled domain also contains context sensors embedded in the home environment. One type of context sensors is used to measure physical properties of the environment such as temperature, humidity and air quality. Another type of sensors can be used to determine the presence, location, and activity of the residents, and be used in combination with software applications such as an electronic agenda to provide supplementary information for reasoning.

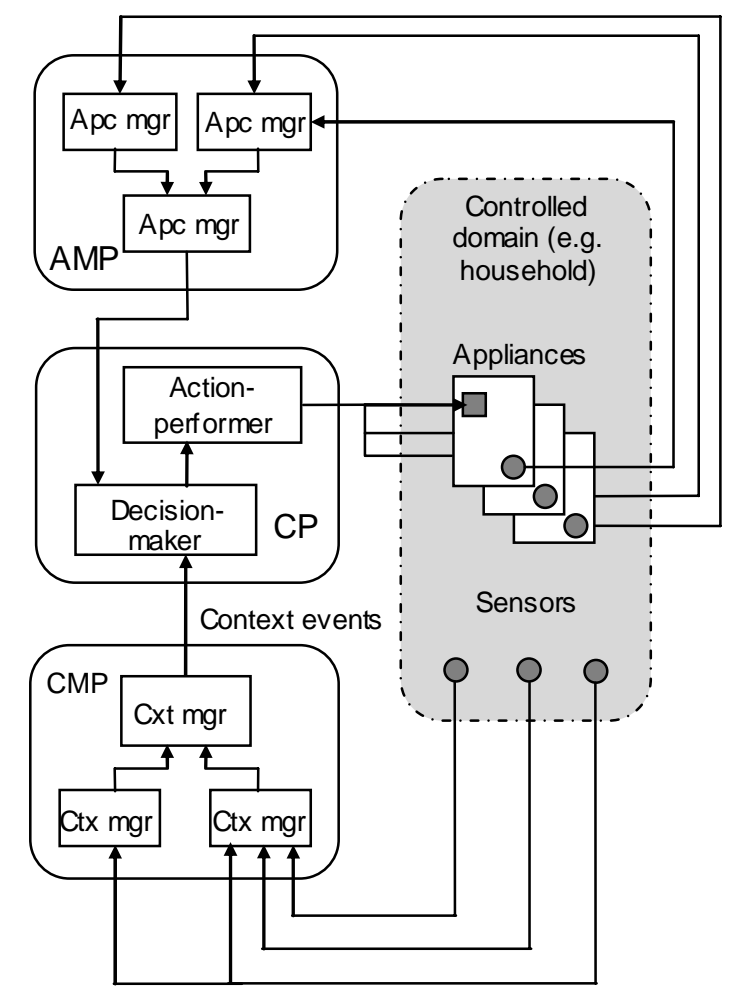

Fig. 1. Basic architecture for household energy management (adapted from [48]).

The monitored energy consumption of several appliances may be collected by an appliance manager (Apc mgr in Fig. 1) in order to get useful insights and derive consumption aggregates for certain categories of appliances. Multiple appliance managers may be organized in a tree structure with a root/top appliance manager that produces the right-level consumption information on basis of which control decisions 
can be made. The coordination of tasks of appliance managers is called the Appliances Management Process (AMP).

Similarly, raw context data of one or more context sensors may be collected by a context manager (Cxt mgr in Fig. 1) in order to derive more reliable or higher-level context information. Through a process of context reasoning involving one or more context managers the right-level context information is derived which can be used for taking control decisions. The coordination of tasks of context managers is called the Context Management Process $(C M P)$.

Both the results of the $A M P$ and $C M P$ are used to take control decisions. For example, if the $A M P$ indicates that the consumption is approaching a previously set maximum level, certain appliances may be temporarily switched off. However, if the $C M P$ indicates that residents are in the house then no actions should be taken that would go against a previously set comfort level. Control decisions are based on a set of rules that aim at realizing end-user goals by proposing actions based on a view of the currently evolving situation. End-user goals are for example reducing energy consumption and maintaining a certain comfort level. The view of the situation is based on information received from the AMP and CMP. Possibly also learned patterns can be considered. If an evolving situation matches such a pattern, the actions defined for that pattern will be proposed. Taking control decisions is the responsibility of a component called Decision-maker. Another component, called Action-performer, translates the proposed actions into operations to be performed on selected appliances. For example, the Action-performer may send a request to the actuator of the freezer to temporarily power down.

\section{Basic Service-oriented Architecture}

The basic architecture of the household energy management system identifies components and their interconnection structure but it does not consider interoperability and flexibility issues. One can expect that devices for monitoring and control will be offered by many different vendors. These devices will have widely varying capabilities and use different communication solutions, depending on the type of appliance or environment in which they will be embedded. A similar reasoning applies to software components, which may employ many different algorithms for reasoning and decision making. Developing a dedicated interoperability solution for a given set of heterogeneous components is hard, and most likely results in a system with no or limited possibilities to change the interconnection structure, replace or add components, and use components' functions in various combinations and orders. The latter may be an important drawback if the system is to be deployed in different types of homes and households and has to be maintained under evolving user requirements and technology developments.

We adopt a service-oriented architecture in order to cope with the problems of heterogeneity and rigidity [20,37]. This means, among others, that we assume that components expose their functionality through service interfaces, and can be accessed accordingly using applicable Web service standards [38]. Proprietary solutions should 
then be wrapped such that functionality is appropriately translated between standardbased public service interfaces and internal technology-specific interfaces.

\subsection{Overview of Services}

We initially identify the following services in our basic service-oriented architecture for a household energy management system:

- Consumption (Csn) sensor service: This is a service provided by a (wrapped) sensor attached to or embedded in a household appliance for measuring energy consumption. It supports either a notification or a request-response message exchange. In the former case a notification is sent to interested clients at regular intervals or in case of relevant events. The notification contains measurement values. Relevant events may be raised on crossing consumption thresholds or starting/ending thermostatically triggered activity cycles. The request-response message exchange is initiated by interested clients, which send a request to the service and ask measurement values to be returned in a response.

- Power actuator (Pwr actr) service: This is a service provided by a (wrapped) actuator attached to or embedded in a household appliance for power management. This means that the actuator can be instructed to perform a power on/off operation or switch to another power mode. The service supports a oneway message exchange with which a client can invoke one of the defined operations on the service.

- Context (Cxt) sensor service: This is a service provided by a (wrapped) sensor embedded in the home environment for measuring a context attribute. It supports either a notification or a request-response message exchange. In the former case a notification is sent to interested clients at regular intervals or in case of relevant events. The notification contains measurement values or a value identifying the event type. Relevant events may be raised on crossing context attribute value thresholds such as a critical temperature, or a step change in the context attribute value such as a person entering a room. The request-response message exchange is initiated by interested clients, which send a request to the service and ask measurement values or event indications to be returned in a response.

- Appliance Management Process (AMP) service: This service provides consumption information to the $C P$ service (see below). For this purpose it supports either a notification or a request-response message exchange, to notify the $A P$ service or to respond to a request from the $A P$ service, respectively. The consumption information passed to the $A P$ service has the right scope and content to take control decisions. The $A M P$ service is also a client of one or more Csn sensor services. Using the latter services, it collects measurements values concerning energy consumption of controlled appliances, which it uses to derive the higher-level consumption information aggregates.

- Context Management Process (CMP) service: This service provides context information to the $C P$ service (see below). For this purpose it supports either a notification or a request-response message exchange, to notify the $A P$ service or to respond to a request from the $A P$ service, respectively. The context 
information has the right semantic level to take control actions. The CMP service is also a client of one or more Cxt sensor services. Using the latter services, it collects measurement values or event notifications concerning the context of the residents, which it uses to derive the higher-level context information aggregates.

- Control Process $(C P)$ service: This service provides an interface to the end-users for programming the household energy management system. Programming may consist of specifying simple workflows or rules, or choosing between alternative pre-defined workflows or rules and possibly supplying values for parameters. The $C P$ service is a client of both the $A M P$ service and the CMP service, which provide the necessary information to take control decisions. In addition, it is a client of one or more Pwr actr services, which can be instructed to perform certain operations in accordance to the decisions.

Fig. 2 illustrates the basic service-oriented architecture, using an ad-hoc notation to distinguish between client ports (to invoke operations on an external service) and service ports (to offer operations which can be invoked by external clients).

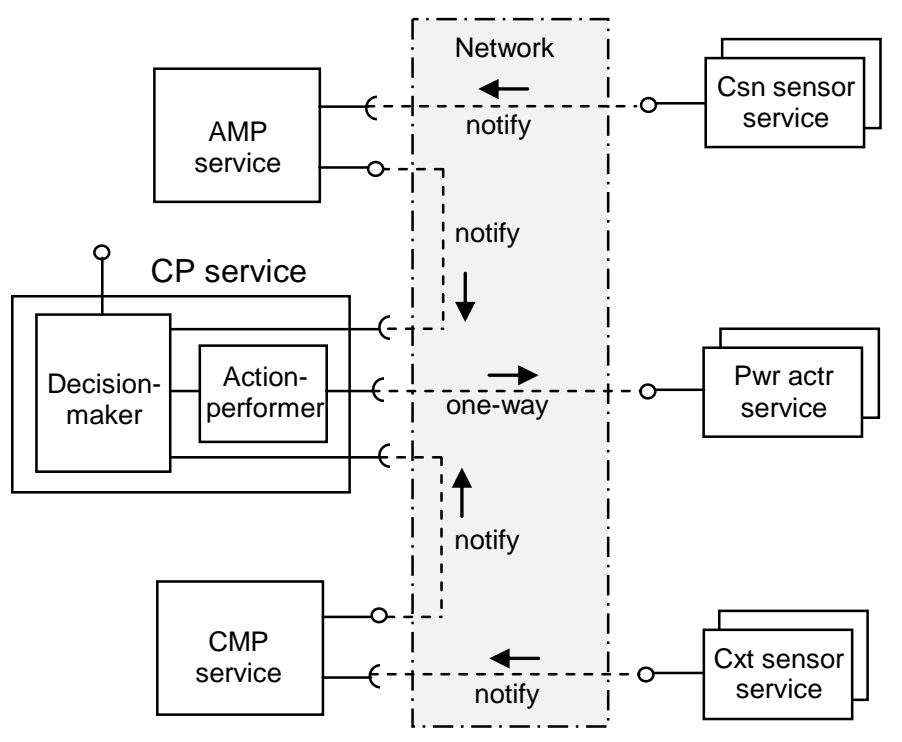

Fig. 2. Basic service-oriented architecture.

\subsection{Service Granularity and Service Hierarchies}

According to the basic system architecture described in the previous section, $A M P$, $C M P$ and $C P$ are processes each involving (potentially) multiple components. This 
brings us to the question whether we want to consider the services corresponding to these processes as individual services or as composite services. Each 'process' service may either be provided by an integrated implementation of the components identified in the process or it may be provided by an orchestration of 'component' services. To answer this question one has to consider what is the right granularity of services [27].

We foresee that in case the controlled domain is a single household, there is no need to have the processes implemented as an orchestration of separate services corresponding to the identified components. The reason for this is that the processes are probably not physically distributed and there is no opportunity to reuse the functionality of the components as services. On the other hand, in case the controlled domain covers a larger geographical area such as an apartment building or a city block, which contains a large number of controlled appliances, the use of composite services might be a good idea.

Several alternatives exist to structure the household energy management system in terms of services when we consider multiple levels or hierarchical domains of energy management. The motivation for considering this is twofold. First of all, by having a system that can coordinate individual household energy management systems, more opportunities exist to avoid peaks and to balance demand and supply (in case of micro-generation). This is particularly interesting to the local power company. Secondly, by organizing households into a collective, leverage is created for individual households to negotiate better prices.

We briefly explore two alternatives for coordinating individual household management systems. These alternatives are not necessarily optimal or practical, but illustrate the spectrum of possibilities for coordination. We defer a more thorough treatment of this topic as future work. Let us assume we have two levels of energy management, say an apartment level and an apartment building level. The first alternative assigns to the highest level considerable responsibility and authority for direct control based on the overall situation (see Fig. 3):

- Each apartment has a single appliance manager and a single context manager, which report to an appliance manager and a context manager at the apartment building level, respectively. The top-level managers inform the $C P$ at the apartment building level. This $C P$ has a decision-maker that analyzes consumption and context, and makes decision proposals. The decision proposals are forwarded to the apartment building's action-performer, which propagates decision proposals in an appropriate way to action-performers at the apartment level.

The second alternative assigns to the highest level no responsibility and authority for direct control, but instead allows changing the policy for control based on the overall situation (see Fig. 4):

- Each apartment has its own AMP and CMP, which report to the apartment's CP. Each apartment's $C P$ makes its own analysis with the decision-maker and controls its own appliances accordingly with the action-performer. However, each apartment's $C P$ also forwards the notifications of the $A M P$ and $C M P$ (or less frequent summary reports) to the $C P$ at the apartment building level. The latter $C P$ has another task and structure than the CPs at the apartment level. It analyzes the consumption and context information with the objective to decide 
whether criteria for decision rules at the apartment level should be modified. If so, it informs selected CPs at the apartment level of the new criteria.

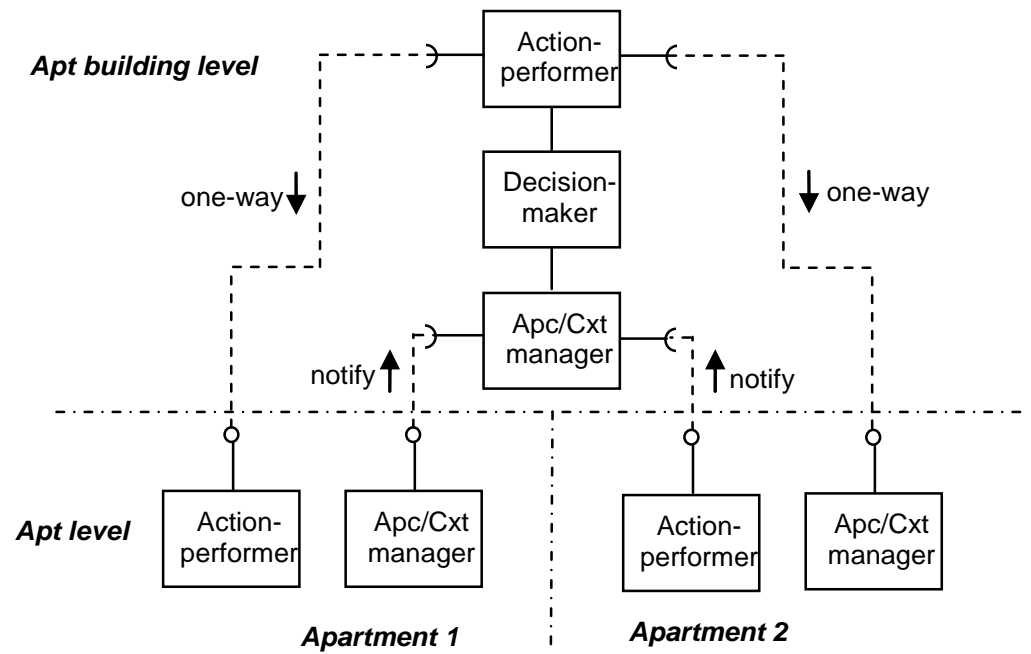

Fig. 3. Multi-level energy management - alternative 1 .

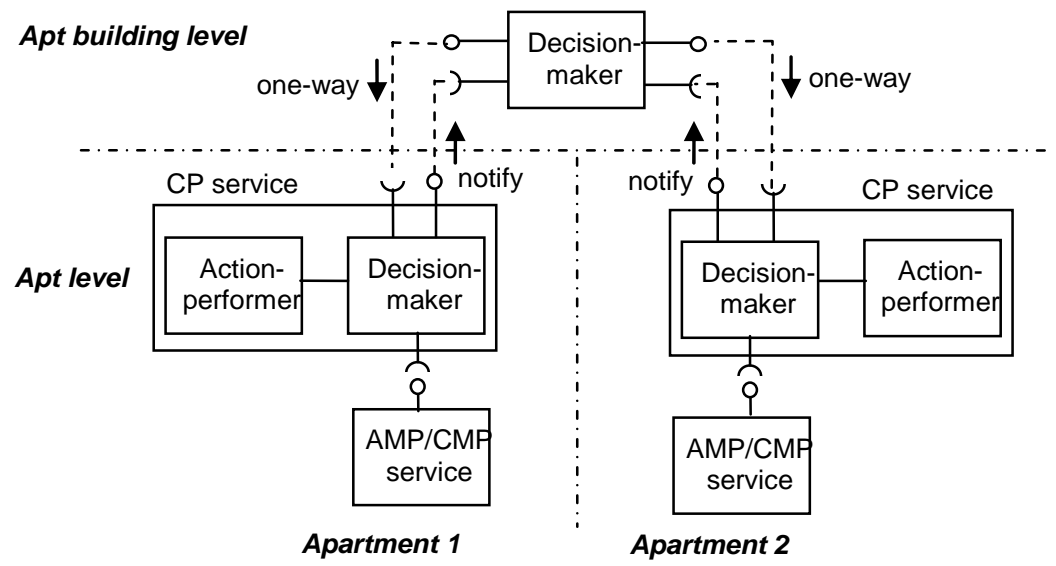

Fig. 4. Multi-level energy management - alternative 2.

The second alternative resembles a reflective architecture as has been proposed for some middleware systems [9]. A reflective system offers a separation of concerns and provides inspection and adaptation of its own behavior [32]. These properties would 
facilitate dynamic adaptation [6] and self-healing [35], as is generally required in wireless settings with mobile and context-aware applications.

In our case of household energy management, two meta-levels of reflection can be recognized: (i) at the first level, appliances are monitored, and decisions are made to change the consumption behavior of appliances based on some management policy model; and (ii) at the second level, households are monitored, and decisions are made to change the energy management policy of households based on some performance goal model. Fig.5 shows these levels. In this paper, we will not further explore the exploitation of reflection for our service-oriented architecture, but leave this for future work.

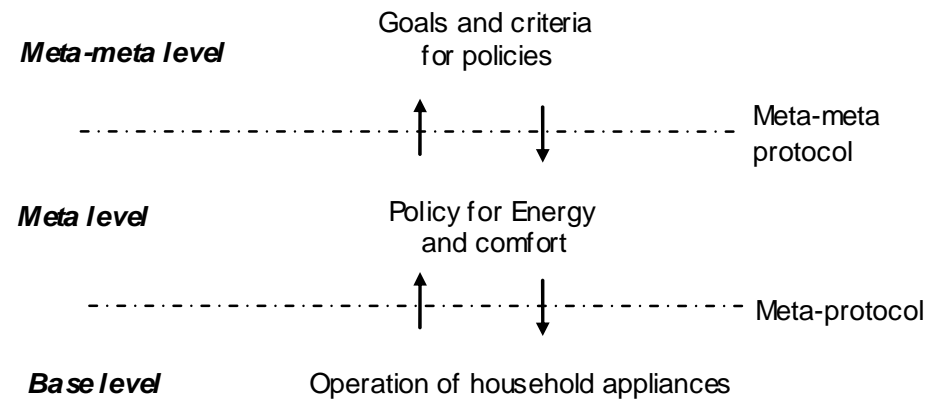

Fig. 5. Reflective levels in energy management.

\section{Goal-driven Control Process}

The control process that provides the $C P$ service and comprises the Decision-maker and Action-performer (see Fig. 2) is driven by end-user goals and realized by the execution of a rule-based policy that fulfils these goals. The overall goal that we project is a combination of energy and comfort conservation. Since the components in this goal are to some extent conflicting, and their weights may be case-specific as well as dependent on the stakeholders, balancing these components is very challenging. We can distinguish between two stakeholder types: the resident or house owner, typically the consumer of energy (if we disregard micro-generation), and the power company, the main producer of energy. Their interests can be characterized as follows (see for example also [54]):

- Producer: (i) keep peaks below what can be supported, as power outages and power quality fluctuations lead to consumer complaints; (ii) minimize overcapacity and avoid large variations in time, as this would otherwise require expensive dimensioning of the grid; and (iii) minimize supply and waste, for environmental reasons.

- Consumer: (i) minimize local consumption, for economic and environmental reasons; and (ii) optimize local comfort, for consumer-selfish reasons. 
Underlying the rule-based policy is a knowledge representation that allows the selection and evaluation of rules. We do not discuss here the requirements that hold for this knowledge representation and how the knowledge base can be up to date. For this the interested reader is referred to [52]. Instead, we end this section with some (loosely formulated) examples of rules that could be used by a control process:

1. If a room is not used, then any device in the room from the list (light, heating, air conditioning, entertainment equipment, etc.) can be switched off, unless somebody has indicated that it should stay on.

2. During low consumption periods, battery-powered devices (such as electric cars, household robots, battery-powered appliances and tools) can be charged as far as they need charging.

3. During peak consumption periods, thermostatically controlled devices in the house (such as room heating, air conditioning, refrigerator, freezer) can be operated in interleave or low-power mode.

\section{Home Service Bus}

The service-oriented architecture depicted in Fig. 2 comprises a network component that provides the connection infrastructure for the services. We assumed that this component is capable of realizing the message exchange between service requestors (clients) and service providers (services), using available network technologies. However, we may attribute additional generic functionality to this infrastructure, thus relieving individual service developers from the task of implementing such functionality over and over again. This idea is similar to that of enterprise service bus $[33,46]$, and we therefore call this infrastructure the home service bus. Presumably, a home service bus will have similar functionality as an enterprise service bus, but would favor different implementations because of the specific requirements of home applications and the characteristics of home networks.

The home service bus plays a key role in realizing the service-oriented architecture. First of all, it can provide the register-find-bind functionality which is one of the central promises of service-oriented architecture [10, 20]. And secondly, it can provide a virtual operational environment to service requestors. We will briefly expand on these functions.

The home service bus manages a service directory which contains relevant information on services. The information on a service is added to the directory if the service is registered. This can be done automatically, when the service is deployed, or after deployment when it is discovered by the bus. The information covers both application aspects of the service as well as network aspects of the service endpoint. A service requestor can find a service it is interested in by enquiring the directory. The directory uses the application related information to match registered services with capabilities required by the service requestor, and selects the best service from the qualified ones by using run-time operational information. The service requestor can then bind to the selected service using the network information on the service endpoint. 
Effectively, a service requestor can find and subsequently use a service without needing to know the technical details of this service and that the service may be just one of many qualified services. The set of qualified services represents the virtual service in which the service requestor is interested. The home service bus hides internal implementation details of the services, such as the used programming language, runtime environment and hardware platform, but also allows the service requestor to interact with the selected service without knowledge of the network address or the communication protocol. Several kinds of virtualization or mediation functions may be provided by the service bus in order to cope with the heterogeneity of service implementations. For example, wrapping and transformation functions are needed if peer components do not share a common communication protocol or message format, as can be expected in the heterogeneous home environment. Some services may reside on a home gateway or server system, where Web services for communication (SOAP) and description (WSDL) can be supported. However, resource-limited devices, such as sensors and actuators, have to use product type specific industry standards. Therefore, the home service bus has to support such standards, and internally apply proper interface wrapping and/or protocol translation in order to make these devices appear as web-enabled devices to components that natively support web services. Additional functions are also possible, including but not limited to:

- $\quad$ Routing: Messages may be routed to a particular system in case of error situations, or to a preferred service or set of services based on workload or availability. For example, service selection for the virtual service may be done on a per-request basis, using request-time operational and possibly context information.

- Publish/subscribe: Service requestors may subscribe to certain responses or events with a single subscribe request to the service bus. The service bus then takes care of notifying the requestor of responses or events according to the specification in the subscribe request, until the subscription is withdrawn. For example, energy consumption measurement values or events from consumption sensors can be delivered this way to appliance management processes.

- Booking: Service requestors may book certain services which are not immediately available or not immediately needed. The service bus schedules provisioning of booked services to requestors based on priorities or overall performance criteria. For example, energy supply can be considered as a service and smart appliances may request certain energy-hungry tasks to be performed, but leave the timing to the bus.

Fig. 6 shows the discussed functionality of the home service bus as infrastructure services. 


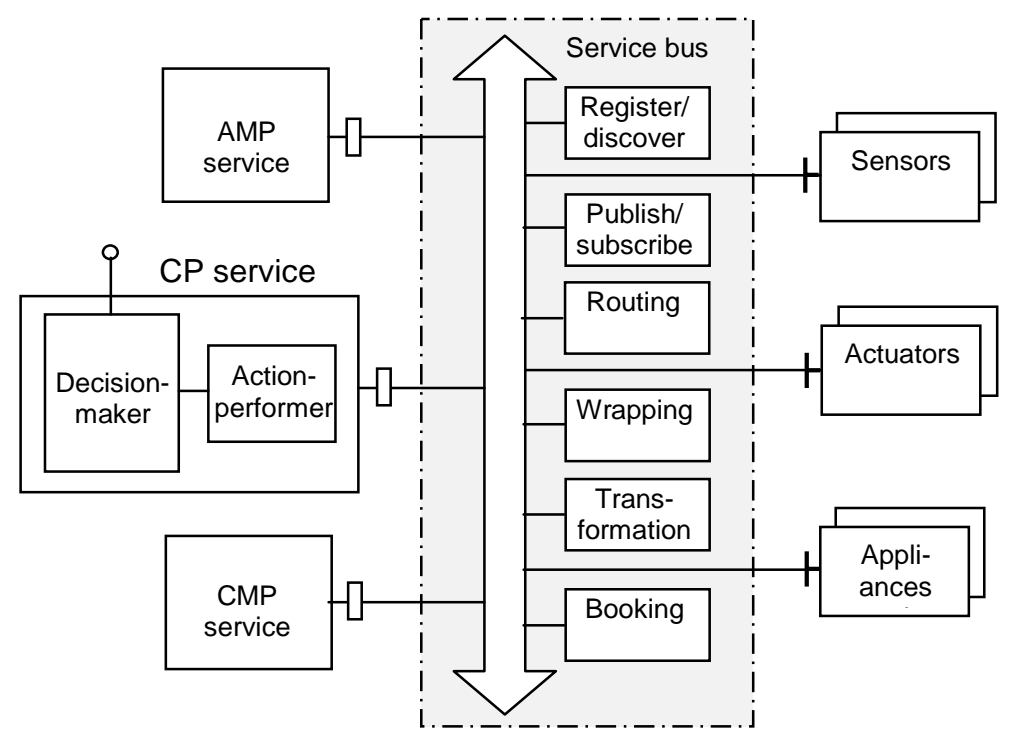

Fig. 6. Home service bus as mediator between services relevant for household energy management.

It is interesting to consider how the home service bus can be realized using various existing industry standards, and what alternatives exist for connection infrastructures that support service-oriented architecture. Relevant standards include Open Service Gateway initiatibe (OSGi), European Installation Bus (EHS), Home Audio Video interoperability (HAVi), Universal Plug and Play (UPnP), and Jini. In particular, several projects have explored the use of OSGi in combination with other standards for the smart home [50, 40, 56, 25].

Another interesting question concerns the right functionality of the home service bus, considering the different application areas that exist for the smart home domain. Many research projects have focused on the development of an network infrastructure or middleware for the smart home with one specific application area in mind, such as infotainment and home automation [41], elderly care and independent living [51, 36, 22], and energy management [29, 47]. This led to different proposed solutions. However, it is unlikely that multiple home service buses will co-exist in the same home. The problem is that some functionality may be generic in one application area, but may not be used in another area. Another problem is that different application areas will have their own industry specific standards. Finding a cost-effective compromise on which functions to include and which standards to support is therefore an important challenge. 


\section{Conclusion}

Household energy consumption accounts for a significant portion of most nations' overall consumption. It is therefore worthwhile to investigate possibilities of reducing energy consumption of households through effective energy management. To be effective, household energy management systems should promote energy conscious behavior of end-users. This is not easy to achieve, since we still know little about the behavior of end-users. Some studies have shown that providing immediate feedback to residents on consumption and related costs can have a positive impact on user behavior and lead to considerable energy savings. Advances in pervasive computing have enabled smart home applications, with energy management as one of the targeted application areas. For example, this development led to affordable technical solutions for providing immediate feedback through smart metering and advanced user interfaces. Smart home applications for energy management can also be used to automate part of the energy conscious behavior of people, namely by making such applications responsible for realizing comfort and consumption (c.q. reduction) targets that have been formulated by the residents.

Automated systems to support household energy management consist of heterogeneous components, are based on various industry standards, and are subject to evolving user requirements and technology developments. We claim that adoption of a service-oriented architecture for household energy management systems is particularly useful to cope with interoperability and flexibility issues. We illustrate the service-oriented approach for a system that is able to monitor both the consumption of household appliances and the presence and activity of residents, and that accordingly takes 'smart' decisions on when and where to intervene in the consumption of appliances. We further discuss the role of a home service bus to realize some of the key properties of a service-oriented architecture.

Several challenges and topics for future work have been identified. One major challenge is the federation of multiple household energy management systems in order to exploit economies of scale. The federated system would have two or more hierarchical levels of management, and it is as yet unclear which architecture would best support multi-home energy conservation opportunities while sufficiently ensuring autonomy of individual households. We also like to extend our household energy management system with micro-generation. Especially, it is interesting to consider the possibility of dynamically matching demand and supply of energy in combination with buying energy from and selling energy to the electricity grid. Another major challenge is finding an acceptable architecture and technical configuration of the home service bus such that it can act as the service-oriented backbone for all application areas in the smart home.

\section{References}

1. Aarts, E., Wichert, R.: Ambient Intelligence. In: Technology Guide, pp. 244-249. Springer (2009) 
2. Abrahamse, W., Steg, L., Vlek, C., Rothengatter, T.: A Review of Intervention Studies Aimed at Household Energy Conservation. Journal of Environmental Psychology 25: 273-291 (2005)

3. Ahuja, S., Potti, P.: An Introduction to RFID Technology. Communications and Network 2 (3): 183--186 (2010)

4. Allen, S.R., Hammond, G.P., McManus, M.C.: Prospects for and Barriers to Domestic Micro-generation: A United Kingdom Perspective. Applied Energy 85 (6): 528--544 (2007)

5. Burgess, J., Nye, M.: Re-materialising Energy Use through Transparent Monitoring Systems. Energy Policy 36: 4454--4459 (2008)

6. Capra, L., Emmerich, W., Mascolo, C.: CARISMA: Context-aware Reflective Middleware for Mobile Applications. IEEE Transactions on Software Engineering 29 (10): 929--945 (2003)

7. Chetty, M, Tran, D., Grinter, R.E.: Getting to Green: Understanding Resource Consumption in the Home. In: 10th International Conference on Ubiquitous Computing, UbiComp, pp. 242--251. ACM Press (2008)

8. Cook, D.J., Youngblood, M., Das, S.K.: A Multi-agent Approach to Controlling a Smart Environment. In: Designing Smart Homes - The Role of Artificial Intelligence, pp. 165-182. LNCS 4008, Springer-Verlag (2006)

9. Costa, F.M., et al.: The Role of Reflective Middleware in Supporting the Engineering of Dynamic Applications. In: Reflection and Software Engineering, pp. 79--98. LNCS 1826, Springer-Verlag (2000)

10.Curbera, F. et al.: Unraveling the Web Services Web - An Introduction to SOAP, WSDL, and UDDI. IEEE Internet Computing 6 (2): 86--93 (2002)

11.Darby, S.: The Effectiveness of Feedback on Energy Consumption - A Review for DEFRA of the Literature on Metering, Billing and Direct Displays. Environmental Change Institute, University of Oxford (2006)

12.Darby, S.: Smart Metering: What Potential for Householder Engagement? Building Research \& Information 38 (5): 442--457 (2010)

13.Dey, A.K.: Understanding and Using Context. Personal and Ubiquitous Computing Journal 5 (1): 4--7 (2001)

14.Davidoff, S., et al.: Principles of Smart Home Control. In: 8th International Conference on Ubiquitous Computing, UbiComp, pp. 19--34. LNCS 4206, Springer-Verlag (2006)

15.De Silva, L.C., Methew, S.: Energy Efficient Smart Homes. In: 1st International Conference of the IET Brunei Darussalam Network, pp. P82: 1--4 (2008)

16.DEFRA - Department for Environment, Food and Rural Affairs: Climate Change: Taking Action (2010), http://www.defra.gov.uk/environment/climate/documents/taking-action.pdf

17.Dockhorn Costa, P., Ferreira Pires, L., Sinderen, M. van: Designing a Configurable Platform for Mobile Context-aware Applications. International Journal of Pervasive Computing and Communications 1 (1): 13--25 (2005)

18.Edwards, W.K., Grinter, R.: At Home with Ubiquitous Computing: Seven Challenges. In: International Conference on Ubiquitous Computing, UbiComp, pp. 256--272. LNCS 2201, Springer-Verlag (2001)

19.Energy Information Administration: Reginal Energy Profile - U.S. Household Electricity Report (2005), http://www.eia.doe.gov/emeu/reps/enduse/er01_us.html

20.Erl, T.: Service-oriented Architecture: Concepts, Technology, and Design. Prentice Hall (2005)

21.Estrin, D., Culler, D., Pister, K., Sukhatme, G.: Connecting the Physical World with Pervasive Networks. Pervasive Computing 1 (1): 59--69 (2002)

22.Farella, E., Falavigna, M., Ricco, B.: Aware and Smart Environments: The Casattenta Project. Microelectronics Journal 41 (11): 697--702 (2010)

23.Fischer, C.: Feedback on Household Electricity Consumption: A Tool for Saving Energy? Energy Efficiency 1: 79--104 (2008) 
24.Geller, H. et al.: Policies for Increasing Energy Efficiency: Thirty Years of Experience in OECD Countris. Energy Policy 34 (5): 556--573 (2006)

25.Gouin-Vallerand, C., Giroux, S.: Management and Deployment of Applications with OSGi in the Context of Smart Homes. In: 3rd International Conference on Wireless and Mobile Computing, Networking and Communications, WiMob. IEEE Computer Society (2007)

26.Gustavsson, R.: Agents with Power. Communications of the ACM 42 (3): $41--47$ (1999)

27.Haesen, R., Snoeck, M., Lemahieu, W., Poelmans, S.: On the Definition of Service Granularity and Its Architectural Impact. In: Advanced Information Systems Engineering, pp. 375--389. LNCS 5074, Springer-Verlag (2008)

28.Holroyd, P., Watten, P., Newbury, P.: Why Is My Home Not Smart? In: 8th International Conference on Smart Homes and Health Telematics, ICOST, pp. 53--59. LNCS 6159, Springer-Verlag (2010)

29.Inoue, M., Higuma, T., Ito, Y., Kushiro, N., Kubota, H.: Network Architecture for Home Energy Management System. IEEE Transactions on Consumer Electronics 49 (3): 606--613 (2003)

30.Jenny, A., Díaz López, J.R., Mosler, H.-J.: Household Energy Use Patterns and Social Organisation for Optimal Energy Management in a Multi-user Solar Energy System. Progress in Photovoltaics: Research and Applications 14: 352--362 (2006)

31.Jiang, L., Liu, D.-Y., Yang, B.: Smart Home Research. In: 3rd International Conference on Machine Learning and Cybernetics, pp. 659--663 (2004)

32.Kon, F., Costa, F., Blair, G., Campbell, R.H.: The Case for Reflective Middleware. Communications of the ACM 45 (6): 33--38 (2002)

33.Leymann, F.: Web Services: Distributed Applications without Limits. In: Database Systems for Business, Technology and Web, BTW (2003)

34.Long Ha, D., Ploix, S., Zamai, E., Jocomino, M.: A Home Automation System to Improve Household Energy Control. In: 12th IFAC Symposium on Information Control Problems in Manufacturing. International Federation on Automatic Control (2006)

35.Mei, H., Huang, G., Tsai, W.-T.: Towards Self-healing Systems via Dependable Architecture and Reflective Middleware. In: $10^{\text {th }}$ IEEE International Workshop on Objectoriented Real-time Dependable Systems, WORDS, pp. 337--344 (2005)

36.Mikalsen, M., et al.: Interoperability Services in the MPOWER Ambient Assisted Living Platform. In: 22nd International Congress of the European Federation for Medical Informatics - Medical Informatics in a United and Healthy Europe, pp. 366--370. IOS Press (2009)

37.O’Brien, L., Merson, P., Bass, L.: Quality Attributes for Service-oriented Architectures. In: International Workshop on Systems Development in SOA Environments, SDSOA (2007)

38.Papazoglou, M.P.: Web Services: Principles and Technology. Pearson Prentice Hall (2008)

39.Prekop, P., Burnett, M. Activities, Context and Ubiquitous Computing. Computer Communications 26 (11): 1168--1176 (2003)

40.Ricquebourg, V. et al.: The Smart Home Concept: Our Immediate Future. In: 2006 1st IEEE International Conference on e-Learning in Industrial Electronics, pp. 23--28. IEEE Press (2006)

41.Röcker, C., Janse, M.D., Portolan, N., Streitz, N.: User Requirements for Intelligent Home Environments: A Scenario-driven Approach and Empirical Cross-cultural Study. In: 2005 Joint Conference on Smart Objects and Ambient Intelligence - Innovative Context-aware Services: Usages and Technologies, pp. 111--116. ACM (2005)

42.Rogers, Y.: Moving on from Weiser's Vision of Calm Computing: Engaging Ubicomp Experiences. In: 8th International Conference on Ubiquitous Computing, UbiComp, pp. 404-421. LNCS 4206, Springer-Verlag (2006)

43.Roy, A., Das, S.K., Basu, K.: A Predictive Framework for Location-aware Resource Management in Smart Homes. IEEE Transactions on Mobile Computing 6 (11): 1270--1283 (2007) 
44.Saha, D., Mukherlee, A.: Pervasive computing: A paradigm for the 21st century. IEEE Computer, 36 (3): 25--31 (2003)

45.Satyanarayanan, M.: Pervasive Computing: Vision and Challenges. IEEE Personal Communications 8 (4): 10--17 (2001)

46.Schmidt, M.-T., Hutchison, B., Lambros, P., Phippen, R.: The Enterprise Service Bus: Making Service-oriented Architecture Real. IBM Systems Journal 44 (4): $781--797$ (2005)

47.Shah, N., Tsai, C.-F., Chao, K.-M.: Monitoring Appliances Sensor Data in Home Environment: Issues and Challenges. In: 11th IEEE Conference on Commerce and Enterprise Computing, CEC, pp. 439--444. IEEE Computer Society (2009)

48.Shishkov, B., Warnier, M., Sinderen, M. van: On the Application of Autonomic and Context-aware Computing to Support Home Energy Management. In: 12th International Conference on Enterprise Information Systems, ICEIS. INSTICC (2010)

49.Si, H., Kawahara, Y., Morikawa, H., Aoyama, T.: A Stochastic Approach for Creating Context-aware Services Based on Context Histories in Smart Home. In: 3rd International Workshop on Exploiting Context Histories in Smart Environments (ECHISE) (2005)

50.Valtchev, D., Frankov, I.: Service Gateway Architecture for a Smart Home. IEEE Communications Magazine 40 (4): 126--132 (2002)

51.Wang, F., Turner, K.J.: Towards Personalised Home Care Systems. In: 1st ACM International Conference on Pervasive Technologies Related to Assistive Environments, PETRA. ACM (2008)

52.Warnier, M., Sinderen, M. van, Shishkov, B.: Adaptive Knowledge Representation for a Self-managing Home Energy Usage System. In: 4th International Workshop on Enterprise Systems and Technology, I-WEST. SciTePress (2010)

53.Weiss, R.J., Craiger, J.P.: Ubiquitous Computing. The Industrial-organizational Psychologist 39 (4): 44--52 (2002)

54.Willis, K.G., Garrod, G.D.: Energy Efficiency: Economic Constraints to Environmental Quality. International Journal of Environmental Technology and Management 1 (3): 334-337 (2001)

55.Wood, G., Newborough, M.: Dynamic Energy-consumption Indicators for Domestic Appliances: Environment, Behaviour and Design. Energy and Buildings 35: 821--841 (2003)

56.Wu, C.-L., Liao, C.-F., Fu, L.-C.: Service-oriented Smart-home Architecture Based on OSGi and Mobile-agent Technology. IEEE Transactions on Systems, Man, and Cybernetics-Part C: Applications and Review 37 (2): 193--205 (2007)

57.Xu, N.: A Survey of Sensor Network Applications. IEEE Communications Magazine 40 (8): 102--114 (2002) 